\title{
Alkoholkrankheit
}

\section{Reduktion des Konsums als Therapieansatz}

In der Behandlung alkoholkranker Patienten findet derzeit ein Umdenken statt: Für viele Ärzte und Betroffene stellt das Konzept des reduzierten Trinkens ein wichtiges Zwischenziel auf dem Weg zur Abstinenz dar. In Deutschland würden nur etwa 9\% der 1,3 Millionen akoholabhängigen Patienten behandelt, beklagte Professor Jürgen Rehm, Dresden. Verbessern ließe sich die Versorgungssituation durch intensive Prävention und Früherkennung sowie den Abbau von Behandlungsbarrieren, um mehr Alkoholkranke in therapeutische Einrichtungen zu bekommen. Dies lasse sich mit den derzeit fast ausschließlich abstinenzorientierten Therapieangeboten im klinischen Alltag häufig nicht umsetzen. Für Betroffene, die sich zunächst nicht zu einer abstinenten Lebensweise motivieren lassen, könne die Konsumreduktion ein erster wichtiger Schritt sein.

Erleichtert werden kann der „Einstieg in den Ausstieg" mit dem neuen Opioidsystemmodulator Nalmefen, wie drei

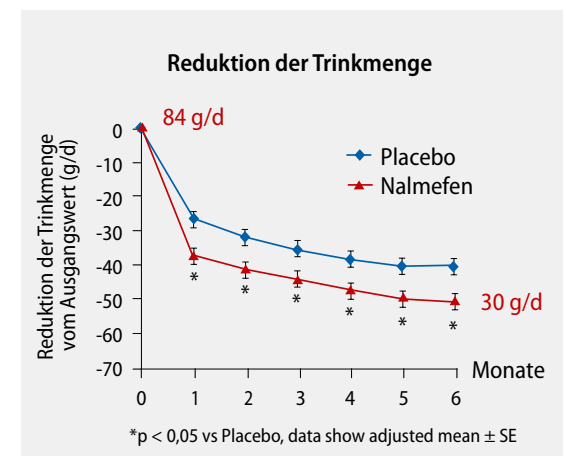

Signifikant stärkere Reduktion des Alkoholkonsums unter Nalmefen versus Placebo

große Phase-III-Studien mit knapp 2.000 alkoholabhängigen Patienten belegen. Als Therapieziele wurden erstmals die Reduktion von Trinkmenge und starken Trinktagen definiert. Wie Professor Karl Mann, Mannheim, berichtete, reduzierte sich der Alkoholkonsum in der Prüf- wie auch in der Kontrollgruppe, unter Verum jedoch signifikant stärker. Nach sechs Monaten war die Trinkmenge um etwa $65 \%$ gesunken (von $84 \mathrm{~g} / \mathrm{d}$ auf $30 \mathrm{~g} / \mathrm{d}$ ) und konnte auch nach einem Jahr noch auf diesem Level gehalten werden. Parallel dazu reduzierte sich die Häufigkeit starker Trinktage (von 19 auf 7). Das Präparat war gut verträglich, unerwünschte Ereignisse wie Schwindel, Schlaflosigkeit und Übelkeit waren meist mild und vorübergehend. Dass zwei Drittel der Studienteilnehmer vorher noch nicht wegen ihrer Alkoholabhängigkeit in Behandlung waren, wertete Mann als Hinweis darauf, dass das Konzept der Konsumreduktion eine praktikable Therapiemöglichkeit darstellt. Die Zulassung von Nalmefen wird für 2013 erwartet.

Dr. Martina-Jasmin Utzt, freie Medizinjournalistin

Satellitensymposium „Einstieg in den Ausstieg: Neue Strategien zur Behandlung der Alkoholabhängigkeit" im Rahmen des DGPPN-Kongresses, Berlin, 22.11.2012. Veranstalter: Lundbeck

\section{Morbus Parkinson}

\section{Lebensqualität als Entscheidungsparameter}

Aspekte der Lebensqualität spielen nicht nur in der Frühtherapie des Morbus Parkinson eine wichtige Rolle. Auch in fortgeschrittenen Stadien darf man die subjektive Befindlichkeit nicht vernachlässigen. Dazu gehören neben dem Faktor Verträglichkeit auch nicht-motorische Symptome der Erkrankung, betonte Universitätsprofessor Werner Poewe von der Neurologischen Klinik der Universität Innsbruck.

Post-hoc-Analysen mit Parkinson-Patienten in einem sehr frühen Stadium zeigen für Rasagilin bei dem Quartil mit dem höchsten UPDRS-Gesamtscore $(\geq$ 25,5; Mittelwert: 32,0) einen Rückgang um rund 7 Punkte. Dies führt für Professor Robert Hauser, Universität von Südflorida in Tampa, zu der Frage, „ob man mit einem Dopaminagonisten ein besseres Ergebnis erhalten hätte“. Die in Dublin vorgestellte 15 -wöchige randomisierte Doppelblindstudie ACTOR bestätigt diese Signale. 109 Patienten (Durchschnittsalter 63 Jahre) mit einem frühem
Morbus Parkinson erhielten Rasagilin $1 \mathrm{mg} / \mathrm{d}$ oder Pramipexol 1,5 mg.

Rasagilin verzeichnete bei ähnlicher klinischer Wirksamkeit deutliche Vorteile im Hinblick auf die Sicherheit und Verträglichkeit gegenüber dem Dopaminagonisten. Insgesamt 5,6\% beendeten unter Rasagilin die Studie wegen intolerabler Nebenwirkungen vorzeitig im Vergleich zu 14,3\% unter Pramipexol. Signifikante Unterschiede zugunsten von Rasagilin wurden bei gastrointestinalen Störungen ( $p=0,0015)$ und Schlafstörungen/Tagesschläfrigkeit $(p=0,0266)$ beobachtet. Ein spezifisches Problem vom Pramipexol waren Libidosteigerungen $(\mathrm{p}=0,028)$. Unter Rasagilin traten signifikant mehr Fälle einer Konjunktivitis $(\mathrm{p}=0,0015)$ auf.

Weitere Erkenntnisse zur Optimierung der Therapie durch eine Add-On-Gabe von Rasagilin, etwa zu einer Basistherapie mit Dopaminagonisten, erwartet man von der ANDANTE-Studie. Damit werde, so Hauser, die Inzidenz von Parkin-
son-Symptomen trotz bestehender Pharmakotherapie und das Risiko Dopaminagonisten-assoziierter Nebenwirkungen verringert und der Bedarf von L-Dopa hinausgezögert.

Poewe verwies darauf, dass Rasagilin neben dem frühen Morbus Parkinson eine wichtige Option auch in fortgeschrittenen Stadien ist, wenn sich beispielsweise erste Wearing-Off-Phänome entwickeln. Darüber hinaus lägen inzwischen zahlreiche Daten vor, nach denen Rasagilin auch nicht-motorische Symptome verringere. Dieser „nicht sichtbare Teil des Eisbergs der Erkrankung" werde oft nicht ausreichend berücksichtigt.

Dr. Alexander Kretzschmar, freier Medizinjournalist

Satellitensymposium „The evolution of treatment decisions in Parkinson's disease" beim $16^{\text {th }}$ International Congress of Parkinson's Disease and Movement Disorders der Movement Disorders Society (MDS), Dublin, 19.6.2012. Veranstalter: TEVA International \& Lundbeck International 